\title{
MAXIMAL NONNEGATIVE AND $\theta$-ACCRETIVE EXTENSIONS OF A POSITIVE DEFINITE LINEAR RELATION
}

\author{
STOROZH O.G.
}

\begin{abstract}
Let $L_{0}$ be a closed linear positive definite relation ("multivalued operator") in a complex Hilbert space. Using the methods of the extension theory of linear transformations in a Hilbert space, in the terms of so called boundary value spaces (boundary triplets), i.e. in the form that in the case of differential operators leads immediately to boundary conditions, the general forms of a maximal nonnegative, and of a proper maximal $\theta$-accretive extension of the initial relation $L_{0}$ are established.

Key words and phrases: Hilbert space, relation, operator, accretive, extension, boundary value space.
\end{abstract}

Ivan Franko National University, 1 Universytetska str., 79000, Lviv, Ukraine

E-mail: storogeukr. net

\section{INTRODUCTION}

The theory of linear relations (multivalued operators) in Hilbert space was initiated by R. Arens [1]. Various aspects of the extension theory of linear relations (in particular, nondensely defined operators; first of all, Hermitian ones) were studied by a number of authors (see, e.g. [2-5] and references therein.

Let us explain that under (closed) linear relation in $H$, where $H$ is a fixed complex Hilbert space equipped with inner product $(\cdot \mid \cdot)$ and corresponding norm $\|\cdot\|$, we understand a (closed) linear manifold in $H^{2} \stackrel{\text { def }}{=} H \oplus H$ and that in the theory of linear relations every linear operator is identified with its graph. Each such relation $T$ has the adjoint $T^{*}$ which is defined as follows

$$
T^{*}=H^{2} \ominus J T\left(=J\left(H^{2} \ominus T\right)\right)
$$

(here and below $\oplus$ and $\ominus$ are the symbols of orthogonal sum and orthogonal complement, respectively; $\forall h_{1}, h_{2} \in H$ we define $\left.J\left(h_{1}, h_{2}\right) \stackrel{\text { def }}{=}\left(-i h_{2}, i h_{1}\right)\right)$.

Through the paper we use the following notations: $D(T), R(T)$, $\operatorname{ker} T$ are, respectively, the domain, range, and kernel of a (linear) relation (in particular, operator) $T$. Some basic definitions and notations are presented below:

$$
\begin{aligned}
& D(T)=\left\{y \in H \mid\left(\exists y^{\prime} \in H\right):\left(y, y^{\prime}\right) \in T\right\}, \\
& R(T)=\left\{y^{\prime} \in H \mid(\exists y \in H):\left(y, y^{\prime}\right) \in T\right\}, \\
& \operatorname{ker} T=\{y \in H \mid(y, 0) \in T\} ;
\end{aligned}
$$


$\widehat{\operatorname{ker}} T=\{(y, 0): y \in \operatorname{ker} T\}$;

if $\lambda \in \mathbb{C}$ then $T-\lambda=\left\{\left(y, y^{\prime}-\lambda y\right) \mid\left(y, y^{\prime}\right) \in T\right\}$;

$\operatorname{ker}(T-\lambda)=\{y \in H \mid(y, 0) \in T-\lambda\}(=\{y \in H \mid(y, \lambda y) \in T\}) ;$

$\widehat{\operatorname{ker}}(T-\lambda) \stackrel{\text { def }}{=}\{(y, \lambda y): y \in \operatorname{ker}(T-\lambda)\} ;$

$T^{-1}=\left\{\left(y^{\prime}, y\right) \in H^{2} \mid\left(y, y^{\prime}\right) \in T\right\} ;$

$1_{X}$ is the identity in $X$;

,$+ \dot{+}$ are the symbols of sum and direct sum in a linear space, respectively.

If $X, Y$ are Hilbert spaces then $(\cdot \mid \cdot)_{X}$ is the symbol of scalar product in $X, \mathcal{B}(X, Y)$ is the set of linear bounded operators $A: X \rightarrow Y$ such that $D(A)=X ; \mathcal{B}(X) \stackrel{\text { def }}{=} \mathcal{B}(X, X)$. If $A_{i}: X \rightarrow$ $Y_{i}(i=1,2)$ are linear operators, then the notation $A=A_{1} \oplus A_{2}$ means $A x=\left(\begin{array}{c}A_{1} x \\ A_{2} x\end{array}\right)$ for every $x \in X$.

Let us recall that a linear relation $T$ in $H$ is said to be nonnegative (denoted $T \geq 0$ ) if $\left(y^{\prime} \mid y\right) \geq 0$ for all $\left(y, y^{\prime}\right) \in T$, positive definite (denoted $T \gg 0$ ) if, in addition, $\inf T \stackrel{\text { def }}{=} \inf \left\{\left(u^{\prime} \mid u\right) \mid\left(u, u^{\prime}\right) \in T,\|u\|=1\right\}>0$, and self adjoint if $T=T^{*}$. The linear relation $T \subset H^{2}$ is said to be $\theta$-accretive one $\left(-\frac{\pi}{2} \leq \theta \leq \frac{\pi}{2}\right)$ if

$$
\forall \widehat{y}=\left(y, y^{\prime}\right) \in T \quad \arg \left(y^{\prime} \mid y\right) \in\left[\theta-\frac{\pi}{2}, \theta+\frac{\pi}{2}\right],
$$

i.e.

$$
\forall \widehat{y} \in T \quad \arg \left(\pi_{2} \widehat{y} \mid \pi_{1} \widehat{y}\right) \in\left[\theta-\frac{\pi}{2}, \theta+\frac{\pi}{2}\right],
$$

where $\pi_{1}, \pi_{2}$ are the orthoprojections $H^{2} \rightarrow H \oplus\{0\}$ and $H^{2} \rightarrow\{0\} \oplus H$, respectively. If, in addition, $T$ has no $\theta$-accretive extensions in $H$ we say that $T$ is a maximal $\theta$-accretive relation. In the case when $\theta=0\left(\theta=\frac{\pi}{2}, \theta=-\frac{\pi}{2}\right)$ the $\theta$-accretive relation is called accretive (dissipative, accumulative) one.

It is well known that a closed relation $T \subset H^{2}$ is a maximal $\theta$-accretive relation if and only if the adjoint relation $T^{*}$ is a (maximal) $-\theta$-accretive one. In particular, a nonnegative relation is a maximal one if and only if it is self adjoint.

In this paper, we assume that the closed linear positive definite relation $L_{0} \subset H^{2}$ is given and we try to establish the general form of its maximal nonnegative and proper maximal $\theta$-accretive extension (the extension $L_{1}$ of $L_{0}$ is said to be a proper one if $L_{0} \subset L_{1}=\overline{L_{1}} \subset L_{0}^{*}$ ).

It is known [4] that there exist (nonnegative) self adjoint extensions $L_{F}$ and $L_{K}$ of $L_{0}$ satisfying the following property:

self adjoint extension $L_{1}$ of $L_{0}$ is nonnegative if and only if

$$
\forall \varepsilon>0 \quad \forall y \in H \quad\left(\left(L_{F}+\varepsilon\right)^{-1} y \mid y\right) \leq\left(\left(L_{1}+\varepsilon\right)^{-1} y \mid y\right) \leq\left(\left(L_{K}+\varepsilon\right)^{-1} y \mid y\right) .
$$

For the case of densely defined operator $L_{0}$, this property was proved by M. Krein [9]. The extensions $L_{F}$ and $L_{K}$ are called the Friedrichs and Neumann-Krein extensions of $L_{0}$, respectively. If $L_{0}$ is positive definite, the first of the latter inequalities holds under $\varepsilon=0$ too. 


\section{PRELIMINARIES}

Assume that $L_{0} \gg 0, L \stackrel{\text { def }}{=} L_{0}^{*}$, and $L_{F}$ is the hard (i.e. Friedrichs) extension of $L_{0}$. It follows from the results proved in [4] that

$$
L=L_{F} \dot{+} \widehat{\operatorname{ker}} L \text {. }
$$

Let us denote by $\mathcal{P}$ the skew projection $L \rightarrow L_{F}$ corresponding to this decomposition.

Definition 1. Let $\mathcal{H}$ be a Hilbert space and $\Gamma_{1}, \Gamma_{2} \in \mathcal{B}(L, \mathcal{H})$. The triple $\left(\mathcal{H}, \Gamma_{1}, \Gamma_{2}\right)$ is called hard boundary value space $(B V S)$ of $L_{0}$ if

i) $R\left(\Gamma_{1} \oplus \Gamma_{2}\right)=\mathcal{H} \oplus \mathcal{H}$;

ii)

$$
\forall \widehat{y}, \widehat{z} \in L \quad\left(\pi_{2} \widehat{y} \mid \pi_{1} \widehat{z}\right)=\left(\pi_{2} \mathcal{P} \widehat{y} \mid \pi_{1} \mathcal{P} \widehat{z}\right)+\left(\Gamma_{1} \widehat{y} \mid \Gamma_{2} \widehat{z}\right)_{\mathcal{H}}
$$

Lemma 1. Hard BVS of $L_{0}$ exists. It may be constructed in the following way

$$
\mathcal{H}^{0}=\widehat{\operatorname{ker}} L, \quad \Gamma_{1}^{0} \widehat{y}=P \pi_{2} \mathcal{P} \widehat{y}, \quad \Gamma_{2}^{0} \widehat{y}=\widetilde{P} \widehat{y},
$$

where $P$ is the orthoprojection $H^{2} \rightarrow \widehat{\operatorname{ker}} L$, and $\widetilde{P}$ is the skew projection $L \rightarrow \widehat{\operatorname{ker}} L$ corresponding to the decomposition (1).

Proof. First of all, note that identifying $(h, 0) \in \mathcal{H} \oplus\{0\}$ and $h \in \mathcal{H}$ we may assume that $\widehat{\operatorname{ker}} L=\operatorname{ker} L$.

i) Suppose that $h_{1}, h_{2} \in \operatorname{ker} L$ and consider the system of equations $\Gamma_{1}^{0} \widehat{y}=h_{1}, \Gamma_{2}^{0} \widehat{y}=h_{2}$, i.e. $P \pi_{2} \mathcal{P} \widehat{y}=h_{1}, \widetilde{P} \widehat{y}=h_{2}$, where $\widehat{y} \in L$. Put

$$
\widehat{y}=\left(L_{F}^{-1} h_{1}, h_{1}\right)+\left(h_{2}, 0\right)\left(\in L_{F} \dot{+} \widehat{\operatorname{ker}} L=L\right) .
$$

We have

$$
\mathcal{P} \widehat{y}=\left(L_{F}^{-1} h_{1}, h_{1}\right) \Rightarrow \pi_{2} \mathcal{P} \widehat{y}=h_{1} \Rightarrow \Gamma_{1}^{0} \widehat{y}=h_{1} ; \quad \widetilde{P} \widehat{y}=\Gamma_{2}^{0} \widehat{y}=h_{2}
$$

ii) Suppose that $\widehat{y}, \widehat{z} \in L$. Since

$$
\widehat{y}=\mathcal{P} \widehat{y}+\widetilde{P} \widehat{y}, \quad \widehat{z}=\mathcal{P} \widehat{z}+\widetilde{P} \widehat{z}, \quad \widetilde{P} \widehat{y}, \widetilde{P} \widehat{z} \in \widehat{\operatorname{ker}} L,
$$

the equalities

$$
\pi_{2} \widetilde{P} \widehat{y}=0, \quad \pi_{2} \widetilde{P} \widehat{z}=0
$$

are fulfilled. Therefore

$$
\begin{aligned}
\left(\pi_{2} \widehat{y} \mid \pi_{1} \widehat{z}\right) & =\left(\pi_{2} \mathcal{P} \widehat{y}+\pi_{2} \widetilde{P} \widehat{y} \mid \pi_{1} \mathcal{P} \widehat{z}+\pi_{1} \widetilde{P} \widehat{z}\right)=\left(\pi_{2} \mathcal{P} \widehat{y} \mid \pi_{1} \mathcal{P} \widehat{z}+\pi_{1} \widetilde{P} \widehat{z}\right) \\
& =\left(\pi_{2} \mathcal{P} \widehat{y} \mid \pi_{1} \mathcal{P} \widehat{z}\right)+\left(\pi_{2} \mathcal{P} \widehat{y} \mid \pi_{1} \widetilde{P} \widehat{z}\right)=\left(\pi_{2} \mathcal{P} \widehat{y} \mid \pi_{1} \mathcal{P} \widehat{z}\right)+\left(P \pi_{2} \mathcal{P} \widehat{y} \mid \widetilde{P} \widehat{z}\right)_{\widehat{k e r} L} \\
& =\left(\pi_{2} \mathcal{P} \widehat{y} \mid \pi_{1} \mathcal{P} \widehat{z}\right)+\left(\Gamma_{1}^{0} \widehat{y} \mid \Gamma_{2}^{0} \widehat{z}\right)_{\mathcal{H}^{0}}
\end{aligned}
$$

The equality (2) (under $\mathcal{H}=\mathcal{H}^{0}, \Gamma_{1}=\Gamma_{1}^{0}, \Gamma_{2}=\Gamma_{2}^{0}$ ) is proved.

It should be noted that the conception of hard BVS (to be more exactly - positive BVS) was initiated in $[6-8,11]$ and found its further development, for example, in $[2,3,5]$. 
Remark 1. $\left(\mathcal{H}^{0}, \Gamma_{1}^{0}, \Gamma_{2}^{0}\right)$ is a $B V S$ of $L_{0}$, i.e.

i)

$$
\forall \widehat{y}, \widehat{z} \in L \quad\left(y^{\prime} \mid z\right)-\left(y \mid z^{\prime}\right) \equiv\left(\pi_{2} \widehat{y} \mid \pi_{1} \widehat{z}\right)-\left(\pi_{1} \widehat{y} \mid \pi_{2} \widehat{z}\right)=\left(\Gamma_{1}^{0} \widehat{y} \mid \Gamma_{2}^{0} \widehat{z}\right)_{\mathcal{H}^{0}}-\left(\Gamma_{2}^{0} \widehat{y} \mid \Gamma_{1}^{0} \widehat{z}\right)_{\mathcal{H}^{0}} .
$$

ii) $\operatorname{ker}\left(\Gamma_{1}^{0} \oplus \Gamma_{2}^{0}\right)=L_{0}$.

Proof. Indeed, let $\widehat{y}, \widehat{z} \in L$. It follows from (1) and (3) that

$$
\begin{aligned}
\left(\pi_{1} \widehat{y} \mid \pi_{2} \widehat{z}\right) & =\left(\pi_{1} \mathcal{P} \widehat{y}+\pi_{1} \widetilde{P} \widehat{y} \mid \pi_{2} \mathcal{P} \widehat{z}+\pi_{2} \widetilde{P} \widehat{z}\right)=\left(\pi_{1} \mathcal{P} \widehat{y}+\pi_{1} \widetilde{P} \widehat{y} \mid \pi_{2} \widehat{z}\right) \\
& =\left(\pi_{1} \widehat{y} \mid \pi_{2} \widehat{z}\right)+\left(\pi_{1} \widetilde{P} \widehat{y} \mid \pi_{2} \widehat{z}\right)=\left(\pi_{1} \widehat{y} \mid \pi_{2} \widehat{z}\right)+\left(\pi_{1} \widetilde{P} \widehat{y} \mid P \pi_{2} \widehat{z}\right) \\
& =\left(\pi_{1} \widehat{y} \mid \pi_{2} \widehat{z}\right)+\left(\Gamma_{2}^{0} \widehat{y} \mid \Gamma_{1}^{0} \widehat{z}\right)_{\mathcal{H}^{0},}{ }^{\prime}
\end{aligned}
$$

and whence using (2) for the case $\left(\mathcal{H}, \Gamma_{1}, \Gamma_{2}\right)=\left(\mathcal{H}^{0}, \Gamma_{1}^{0}, \Gamma_{2}^{0}\right)$ we obtain

$$
\left(\pi_{2} \widehat{y} \mid \pi_{1} \widehat{z}\right)-\left(\pi_{1} \widehat{y} \mid \pi_{2} \widehat{z}\right)=\left(\pi_{2} \mathcal{P} \widehat{y} \mid \pi_{1} \mathcal{P} \widehat{z}\right)-\left(\pi_{1} \mathcal{P} \widehat{y} \mid \pi_{2} \mathcal{P} \widehat{z}\right)+\left(\Gamma_{1}^{0} \widehat{y} \mid \Gamma_{2}^{0} \widehat{z}\right)_{\mathcal{H}^{0}}-\left(\Gamma_{2}^{0} \widehat{y} \mid \Gamma_{1}^{0} \widehat{z}\right)_{\mathcal{H}^{0}} .
$$

Since $\mathcal{P} \widehat{y}, \mathcal{P} \widehat{z} \in L_{F}$ and $L_{F}=L_{F}^{*}$ we conclude that $\left(\pi_{2} \mathcal{P} \widehat{y} \mid \pi_{1} \mathcal{P} \widehat{z}\right)=\left(\pi_{1} \mathcal{P} \widehat{y} \mid \pi_{2} \mathcal{P} \widehat{z}\right)$, consequently (4) holds.

The statement i) is proved. Let us prove ii):

$$
\left(\Gamma_{1}^{0} \oplus \Gamma_{2}^{0}\right) \widehat{y}=0 \Leftrightarrow \Gamma_{1}^{0} \widehat{y}=0, \Gamma_{2}^{0} \widehat{y}=0 \Leftrightarrow \forall \widehat{z} \in L \quad\left(y^{\prime} \mid z\right)-\left(y \mid z^{\prime}\right)=0 \Leftrightarrow \widehat{y} \in L^{*}=L_{0} .
$$

Remark 2. i) $\operatorname{ker} \Gamma_{2}^{0}=L_{F} ; \quad$ ii) $\operatorname{ker} \Gamma_{1}^{0}=L_{0}+\widehat{\operatorname{ker}} L$.

Proof. Item i) follows immediately from the definition.

ii) 1) Let $\widehat{y} \in L_{0}\left(\subset L_{F}\right)$. Then $\mathcal{P} \widehat{y}=\widehat{y} \in L_{0}$. Consequently $\pi_{2} \mathcal{P} \widehat{y} \in R\left(L_{0}\right)=(\operatorname{ker} L)^{\perp}$, so $P \pi_{2} \mathcal{P} \widehat{y}=0$, i.e. $\Gamma_{1}^{0} \widehat{y}=0$.

2) Let $\widehat{y} \in \widehat{\operatorname{ker}} L$. Then $\mathcal{P} \widehat{y}=0$, therefore $\Gamma_{1}^{0} \widehat{y}=0$. The inclusion $L_{0} \dot{+} \widehat{\operatorname{ker}} L \subset \operatorname{ker} \Gamma_{1}^{0}$ is proved.

3) Let us show that in latter inclusion " $\subset$ " may be replaced by " $=$ ". We have

$$
\begin{aligned}
\operatorname{ker} \Gamma_{1}^{0} \cap \operatorname{ker} \Gamma_{2}^{0} & =L_{0}, \\
\operatorname{ker} \Gamma_{1}^{0}+\operatorname{ker} \Gamma_{2}^{0} & =L, \\
L_{0}+\widehat{\operatorname{ker}} L & \subset \operatorname{ker} \Gamma_{1}^{0}, \\
\widehat{\operatorname{ker}} L \cap \operatorname{ker} \Gamma_{2}^{0} & =\{0\}, \\
L_{F}+\widehat{\operatorname{ker}} L & =L .
\end{aligned}
$$

It should be proved that in (6) " $\subset$ " may be replaced by " $=$ ". Let us prove this. Suppose that $w \in \operatorname{ker} \Gamma_{1}^{0} \subset L=L_{F} \dot{+} \widehat{\operatorname{ker}} L$. So we have $w=w_{1}+w_{2}$, where $w_{1} \in L_{F}, w_{2} \in \widehat{\operatorname{ker} L}$, therefore $w-w_{2}=w_{1}$. But $w \in \operatorname{ker} \Gamma_{1}^{0}, w_{2} \in \widehat{\operatorname{ker} L} \subset \operatorname{ker} \Gamma_{1}^{0}$ (see (6)), $w_{1} \in L_{F}$, therefore $w-w_{2} \in \operatorname{ker} \Gamma_{1}^{0} \cap L_{F}=L_{0}$ (see (5)). Thus $w=\left(w-w_{2}\right)+w_{2} \in L_{0} \dot{+} \widehat{\operatorname{ker}} L$. 
Proposition 1. Let $\left(\mathcal{H}, \Gamma_{1}, \Gamma_{2}\right)$ be a BVS of $L_{0}$ such that

i) $\operatorname{ker} \Gamma_{2}=L_{F}$,

ii) $\operatorname{ker} \Gamma_{1}=L_{0}+\widehat{\operatorname{ker}} L$.

Then $\left(\mathcal{H}, \Gamma_{1}, \Gamma_{2}\right)$ is a hard BVS of $L_{0}$, in particular (2) holds.

Proof. Taking into account (3) and (4) we conclude the following:

$$
\begin{aligned}
\left(\pi_{2} \widehat{y} \mid \pi_{1} \widehat{z}\right) & =\left(\pi_{2} \mathcal{P} \widehat{y}+\pi_{2} \widetilde{P} \widehat{y} \mid \pi_{1} \mathcal{P} \widehat{z}+\pi_{1} \widetilde{P} \widehat{z}\right)=\left(\pi_{2} \mathcal{P} \widehat{y} \mid \pi_{1} \mathcal{P} \widehat{z}+\pi_{1} \widetilde{P} \widehat{z}\right) \\
& =\left(\pi_{2} \mathcal{P} \widehat{y} \mid \pi_{1} \mathcal{P} \widehat{z}\right)+\left(\pi_{2} \mathcal{P} \widehat{y} \mid \pi_{1} \widetilde{P} \widehat{z}\right) \\
& =\left(\pi_{2} \mathcal{P} \widehat{y} \mid \pi_{1} \mathcal{P} \widehat{z}\right)+\left(\pi_{1} \mathcal{P} \widehat{y} \mid \pi_{2} \widetilde{P} \widehat{z}\right)+\left(\Gamma_{1} \mathcal{P} \widehat{y} \mid \Gamma_{2} \widetilde{P} \widehat{z}\right)_{\mathcal{H}}-\left(\Gamma_{2} \mathcal{P} \widehat{y} \mid \Gamma_{1} \widetilde{P} \widehat{z}\right)_{\mathcal{H}} \\
& =\left(\pi_{2} \mathcal{P} \widehat{y} \mid \pi_{1} \widetilde{P} \widehat{z}\right)+\left(\Gamma_{1} \mathcal{P} \widehat{y} \mid \Gamma_{2} \widetilde{P} \widehat{z}\right)_{\mathcal{H}}-\left(\Gamma_{2} \mathcal{P} \widehat{y} \mid \Gamma_{1} \widetilde{P} \widehat{z}\right)_{\mathcal{H}} .
\end{aligned}
$$

But $\Gamma_{2} \mathcal{P} \widehat{y}=0, \Gamma_{2} \mathcal{P} \widehat{z}=0$, consequently $\Gamma_{2} \widetilde{P} \widehat{z}=0 ; \Gamma_{1} \widetilde{P} \widehat{y}=0$, consequently $\Gamma_{1} \mathcal{P} \widehat{y}=\Gamma_{1} \widehat{y}$. Thus (2) is proved.

\section{MAIN RESULTS}

Let $L_{0}, L,\left(\mathcal{H}, \Gamma_{1}, \Gamma_{2}\right)$ and $\mathcal{P}$ be as above. We assume below that $A_{1}, A_{2} \in \mathcal{B}(\mathcal{H})$ and

$$
L_{1}\left(\equiv L_{A}\right)=\left\{\widehat{y} \in L: A_{1} \Gamma_{1} \widehat{y}+A_{2} \Gamma_{2} \widehat{y}=0\right\} .
$$

Lemma 2. $L_{1}^{*}$ is a $\theta$-accretive (nonnegative) relation if and only if $-A_{1} A_{2}^{*}$ is a $\theta$-accretive (nonnegative) operator.

Proof. At first let us consider the following situation: the operator $A$, defined by the equality $A\left(h_{1}, h_{2}\right) \stackrel{\text { def }}{=} A_{1} h_{1}+A_{2} h_{2}\left(h_{1}, h_{2} \in \mathcal{H}\right)$, is normally solvable one. In this case (see [12, Lemma 9, p. 182])

$$
L_{1}^{*}=\left\{\widehat{z} \in L \mid \exists h \in \mathcal{H}: \Gamma_{1} \widehat{z}=A_{2}^{*} h, \Gamma_{2} \widehat{z}=-A_{1}^{*} h\right\} .
$$

Whence using (2) and (8) we see that $\theta$-accretivity (nonnegativity) of $-A_{1} A_{2}^{*}$ yields $\theta$-accretivity (nonnegativity) of $L_{1}^{*}$. Indeed,

$$
\begin{aligned}
\forall \widehat{y} \in L_{1}^{*}\left(y^{\prime} \mid y\right) & =\left(\pi_{2} \widehat{y} \mid \pi_{1} \widehat{y}\right)=\left(\pi_{2} \mathcal{P} \widehat{y} \mid \pi_{1} \mathcal{P} \widehat{y}\right)+\left(\Gamma_{1} \widehat{y} \mid \Gamma_{2} \widehat{y}\right)_{\mathcal{H}} \\
& =\left(\pi_{2} \mathcal{P} \widehat{y} \mid \pi_{1} \mathcal{P} \widehat{y}\right)+\left(A_{2}^{*} h \mid-A_{1}^{*} h\right)_{\mathcal{H}} \\
& =\left(\pi_{2} \mathcal{P} \widehat{y} \mid \pi_{1} \mathcal{P} \widehat{y}\right)+\left(-A_{1} A_{2}^{*} h \mid h\right)_{\mathcal{H}},
\end{aligned}
$$

consequently the $\theta$-accretivity (nonnegativity) of $-A_{1} A_{2}^{*}$ implies $\theta$-accretivity (nonnegativity) of $L_{1}^{*}$.

Conversely, assume that $L_{1}^{*}$ is a $\theta$-accretive (nonnegative) relation while $-A_{1} A_{2}^{*}$ does not obey to this requirement. Then due to (8) there exists $\widehat{z} \in L_{1}^{*}$ such that for some $\varepsilon>0$ and for each $\lambda \in \mathbb{C}$ satisfying the inclusion $\arg \lambda \in\left[\theta-\frac{\pi}{2}, \theta+\frac{\pi}{2}\right]$ (for arbitrary $\lambda \geq 0$ ) the inequality

$$
\left|\left(\Gamma_{1} \widehat{z} \mid \Gamma_{2} \widehat{z}\right)_{\mathcal{H}}-\lambda\right| \geq \varepsilon
$$

takes place. It follows from the definition of Friedrichs extension that there exists $\widehat{z}_{\varepsilon} \in L_{0}$ such that

$$
\left(\pi_{2}\left(\mathcal{P} \widehat{z}-\widehat{z}_{\varepsilon}\right) \mid \pi_{1}\left(\mathcal{P} \widehat{z}-\widehat{z}_{\varepsilon}\right)\right)<\frac{\varepsilon}{2} .
$$


Taking into account (2) we obtain

$$
\begin{aligned}
\left(\pi_{2}\left(\widehat{z}-\widehat{z}_{\mathcal{E}}\right) \mid \pi_{1}\left(\widehat{z}-\widehat{z}_{\mathcal{\varepsilon}}\right)\right) & =\left(\pi_{2} \mathcal{P}\left(\widehat{z}-\widehat{z}_{\mathcal{\varepsilon}}\right) \mid \pi_{1} \mathcal{P}\left(\widehat{z}-\widehat{z}_{\mathcal{E}}\right)\right)+\left(\Gamma_{1}\left(\widehat{z}-\widehat{z}_{\mathcal{E}}\right) \mid \Gamma_{2}\left(\widehat{z}-\widehat{z}_{\mathcal{E}}\right)\right)_{\mathcal{H}} \\
& =\left(\pi_{2} \mathcal{P}\left(\widehat{z}-\widehat{z}_{\varepsilon}\right) \mid \pi_{1} \mathcal{P}\left(\widehat{z}-\widehat{z}_{\mathcal{E}}\right)\right)+\left(\Gamma_{1} \widehat{z} \mid \Gamma_{2} \widehat{z}\right)_{\mathcal{H}} .
\end{aligned}
$$

From here, using (9) we can see that $L_{1}^{*}$ may not to be a $\theta$-accretive (nonnegative) relation.

In the general case by virtue of Lemma on triple (see e.g. [10, p. 22]) there exist $C, \widetilde{A}_{1}, \widetilde{A}_{2} \in$ $\mathcal{B}(\mathcal{H})$ such that $\operatorname{ker} C=\{0\}, A_{i}=C \widetilde{A}_{i}(i=1,2)$ and the operator $\widetilde{A} \in \mathcal{B}(\mathcal{H} \oplus \mathcal{H}, \mathcal{H})$ defined by the equality $\widetilde{A}\left(h_{1}, h_{2}\right)=\widetilde{A}_{1} h_{1}+\widetilde{A}_{2} h_{2}$ is normally solvable. To complete the proof it is sufficient to take into account that $-A_{1} A_{2}^{*}$ is $\theta$-accretive (nonnegative) operator if and only if $-\widetilde{A}_{1} \widetilde{A}_{2}^{*}$ is a $\theta$-accretive (nonnegative) operator.

Remark 3. By virtue of Lemma 2 , if $L_{1}$ is a maximal $\theta$-accretive relation, then $-A_{1} A_{2}^{*}$ is a $\theta$-accretive operator, i.e.

$$
\operatorname{Re}\left(e^{i \theta} A_{1} A_{2}^{*}\right) \leq 0
$$

In particular, if $L_{1}$ is a maximal nonnegative operator, then

$$
A_{1} A_{2}^{*} \leq 0
$$

Lemma 3. Suppose that $A_{1}, A_{2} \in \mathcal{B}(\mathcal{H})$. The inequality (10) holds if and only if there exist contraction $K \in \mathcal{B}(\mathcal{H})$ and $C \in \mathcal{B}(\mathcal{H})$ such that

$$
A_{1}=C\left(K-1_{\mathcal{H}}\right), \quad A_{2}=e^{i \theta} C\left(K+1_{\mathcal{H}}\right) .
$$

Proof. Put

$$
C_{1}=\frac{1}{2}\left(A_{1}+e^{-i \theta} A_{2}\right), \quad C=\frac{1}{2}\left(-A_{1}+e^{-i \theta} A_{2}\right) .
$$

Then

$$
A_{1}=C_{1}-C, \quad e^{-i \theta} A_{2}=C_{1}+C .
$$

Inserting (12) into (10) we obtain $\operatorname{Re}\left[\left(C_{1}-C\right)\left(C_{1}^{*}+C^{*}\right)\right] \leq 0$, i.e. $C_{1} C_{1}^{*} \leq C C^{*}$.

Applying mentioned above Lemma on triple we can see that there exists a contraction $K \in$ $\mathcal{B}(\mathcal{H})$ such that $C_{1}=C K$. The latter equality together with (12) implies (11). Conversely, if $K \in \mathcal{B}(\mathcal{H})$ and $\|K\| \leq 1$, then (11) implies (10).

Theorem 1. Assume that $L_{0}$ is a positive definite closed linear relation in $H$ and $\left(\mathcal{H}, \Gamma_{1}, \Gamma_{2}\right)$ is its hard BVS. Each proper maximal $\theta$-accretive extension $L_{1}$ of $L_{0}$ may be given in the form (7), where $A_{1}, A_{2} \in \mathcal{B}(\mathcal{H})$. This extension is maximal $\theta$-accretive one if and only if

$$
\operatorname{Re}\left(e^{i \theta} A_{1} A_{2}^{*}\right) \leq 0, \quad \operatorname{ker}\left(A_{1}-e^{-i \theta} A_{2}\right)=\{0\} .
$$

In particular, $L_{1}$ is maximal nonnegative relation if and only if

$$
A_{1} A_{2}^{*} \leq 0, \quad \operatorname{ker}\left(A_{1}-A_{2}\right)=\{0\} .
$$

Proof. It is proved in $[6-8,11]$ that in the situation, when $L_{0}$ is densely defined operator, $L_{1}$ is maximal $\theta$-accretive if and only if there exists $K \in \mathcal{B}(\mathcal{H})$ such that $\|K\| \leq 1$, and

$$
L_{1}=\left\{\widehat{y} \in L:\left(K-1_{\mathcal{H}}\right) \Gamma_{1} \widehat{y}+e^{i \theta}\left(K+1_{\mathcal{H}}\right) \Gamma_{2} \widehat{y}=0\right\}
$$


Similar arguments show that it is true in a general case. The first assertion of the theorem is proved.

Further, applying mentioned above Lemma on triple, we can see that $A_{1}, A_{2} \in \mathcal{B}(\mathcal{H})$ may be given in the form (11), where $\operatorname{ker} C=\{0\}$, therefore the conditions (13) are fulfilled. Conversely, assume that these conditions are fulfilled. Lemma 3 shows that in this case the relations (7) and (15) are equivalent, therefore $L_{1}$ is a maximal $\theta$-accretive relation.

In addition, under the conditions of Lemma 3 we have

$$
\operatorname{Im}\left(A_{1} A_{2}^{*}\right)=\{0\} \Leftrightarrow \operatorname{Im}\left[C\left(K-1_{\mathcal{H}}\right)\left(K^{*}+1_{\mathcal{H}}\right) C^{*}\right]=\{0\} \Leftrightarrow \operatorname{Im}\left(K-K^{*}\right)=\{0\}
$$

(let us remind that $\operatorname{ker} C=\{0\}$ ). In other words (see Lemma 2) the maximal nonnegativity of the relation (15) is equivalent to the selfadjointness of the contraction $K$, whence it is easy to prove the last assertion of the theorem.

Remark 4. It is easy to prove that

i) if $R(A)=\mathcal{H}$ then the second of the conditions (13) (respectively (14)) may be replaced by $\left(A_{1}-e^{-i \theta} A_{2}\right)^{-1} \in \mathcal{B}(\mathcal{H})$ (respectively $\left(A_{1}-A_{2}\right)^{-1} \in \mathcal{B}(\mathcal{H})$ );

ii) if $\operatorname{dim} \mathcal{H}<\infty$ then each of mentioned conditions may be replaced by the condition $R(A)=\mathcal{H}$;

iii) under the investigation of maximal nonnegativity of $L_{1}$ the second conditions in (14) may be replaced by $\operatorname{ker}\left(A_{1} \pm i A_{2}\right)=\{0\}$.

\section{REFERENCES}

[1] Arens R. Operational calculus of linear relations. Pacific J. Math. 1961, 11 (1), 9-23. doi:10.2140/pjm.1961.11.9

[2] Arlinskii Yu.M. Maximal accretive extensions of sectorial operators. D. Sc. dissertation, Institute of mathematics, National Academy of Sciences of Ukraine, Kyiv, 2000. (in Russian)

[3] Coddington E.A. Self-adjoint subspace extensions of nondensely defined linear operators. Bull. Amer. Math. Soc. 1973, 79 (4), 712-715.

[4] Coddington E.A., de Snoo H.S.V. Positive self-adjoint extensions of positive symmetric subspaces. Math. Z. 1978, 159 (14), 203-214.

[5] Derkach V.A., Malamud M.M. Theory of the Extensions of Symmetric Operators and Boundary Problems. Works of the Institute of Mathematics of the National Academy of Sciences of Ukraine, Kyiv, 2017. (in Russian)

[6] Gorbachuk V.I., Gorbachuk M.L. Boundary Value Problems for Differential-Operator Equations. Kluver Academic Publishers, Dordrecht-Boston-London, 1991. (translation of Naukova Dumka, Kyiv, 1984. (in Russian)) 
[7] Kochubei A.N. On extensions of symmetric operators and symmetric binary relations. Math. Notes 1975, 17 (1), 25-28. (translation of Mat. Zametki 1975, 17 (1), 41-48. (in Russian))

[8] Kochubei A.N. On the extensions of positive definite symmetric operator. Dopov. Akad. Nauk Ukr. SSR 1979, 3, 168-171. (in Ukrainian)

[9] Krein M.G. Theory of selfadjoint extensions of semibounded operators. Mat. Sb. 1947, 20 (14), 431-495. (in Russian)

[10] Lyantse V.E., Storozh O.G. Methods of the Theory of Unbounded Operators. Naukova Dumka, Kyiv, 1983. (in Russian)

[11] Mihajlets V.A. Spectra of operators and boundary value problems. Spectral Analysis of Differential Operators. Nauk. Dumka, Kiev 1980, 106-131. (in Russian)

[12] Pihura O.V., Storozh O.G. A resolvent and conditions of solvability for proper extensions of a linear relation in a Hilbert space. Visnyk of the Lviv Univ. Ser. Mech. Math. 2016, 82, 174-185. (in Ukrainian)

Received 01.03.2020

Сторож О.Г. Максимальні невід'ємні та $\theta$-акретивні розширення додатно визначеного лінійного відношення // Карпатські матем. публ. - 2020. — Т.12, №2. — С. 289-296.

Нехай $L_{0}$ - замкнене лінійне додатно визначене відношення (“багатозначний оператор”) у комплексному гільбертовому просторі. Застосовуючи методи теорії розширень лінійних перетворень у гільбертовому просторі, у термінах так званих просторів граничних значень (граничних трійок), тобто у вигляді, який у випадку диференщіальних операторів приводить безпосередньо до крайових умов, встановлено загальний вигляд максимально невід'ємного та власного максимально $\theta$-акретивного розширення початкового відношення $L_{0}$.

Ключові слова і фрази: гільбертів простір, відношення, оператор, акретивний, розширення, простір граничних значень. 